\title{
Susceptibilidad a penicilina en cepas de Streptococcus pneumoniae aisladas en el Hospital de Puerto Montt, 1995-2003
}

\author{
María Luisa Rioseco Z, Raúl Riquelme 0. \\ Penicillin susceptibility \\ in Streptococcus pneumoniae strains \\ isolated at Puerto Montt Hospital \\ between 1995 and 2003
}

Background: The emergence and dissemination of resistance to penicillin among Streptococcus pneumoniae changed the approach to empiric antimicrobial therapy. Aim: To evaluate the in vitro susceptibility to penicillin in all S. pneumoniae strains isolated in Puerto Montt Hospital between 1995 and 2003. Material and methods: We revised all Microbiology Laboratory files of this period. Identification and antimicrobial susceptibility study methods for S pneumoniae did not change during the study period. Results: Six hundred eighteen S pneumoniae strains were identified. Of these, $66 \%$ came from adults and $48 \%$ from invasive diseases. Only $1.9 \%$ of strains were penicillin resistant and $7.6 \%$ had intermediate sensibility. Strains isolated from children and those isolated from non sterile sources had non significantly higher resistance levels. The susceptibility did not change along the years of the study. Conclusions: Among S pneumoniae strains isolated at Puerto Montt Hospital, the prevalence of penicillin resistance is low (Rev Méd Chile 2005; 133: 175-80).

(Key Words: Drug resistance, microbial; Penicillins; Streptococcus pneumoniae)

Recibido el 23 de diciembre, 2004. Aceptado el 29 de junio, 2005.

Laboratorio de Microbiología y Servicio de Medicina, Hospital Puerto Montt

$E^{1}$ Streptococcus pneumoniae es un agente frecuente en las infecciones del tracto respiratorio. Es el principal agente etiológico de otitis media aguda (OMA) ${ }^{1}$, de alta incidencia en niños y de neumonía adquirida en la comunidad (NAC) en adultos que se hospitalizan ${ }^{2}$.

Correspondencia a: María Luisa Rioseco Z. Seminario s/número, Hospital Puerto Montt. Fax: 65 259180. E mail: mlrioseco@llanchipal.cl
La penicilina (PNC) ha sido el tratamiento de elección para muchas de las infecciones producidas por S pneumoniae, microorganismo que se mantuvo uniformemente susceptible hasta fines de la década 1970-79, en que se reportan las primeras cepas resistentes a este B-lactámico ${ }^{3}$. Desde entonces, se ha documentado un aumento en la frecuencia de cepas resistentes en muchos países, con cifras que oscilan entre 5 y $60 \%{ }^{4}$. Este fenómeno ha determinado que la PNC haya sido 
reemplazada por cefalosporinas de tercera generación e incluso por vancomicina, para el tratamiento empírico de cuadros infecciosos donde el $\mathrm{S}$ pneumoniae es uno de los agentes principales.

Los porcentajes de cepas con resistencia a PNC son muy variables, incluso en distintas áreas geográficas de un mismo país ${ }^{5}$. Esto se podría explicar por la variabilidad en los factores asociados a mayor riesgo para la aparición de resistencia, como son el uso frecuente de antimicrobianos, asistencia a sala cuna, etc. En Chile, los datos publicados de susceptibilidad de S pneumoniae corresponden principalmente a cepas aisladas en Santiago y, dada la variabilidad antes mencionada, parece de gran importancia conocer datos de otras regiones, que permitan tomar decisiones racionales para el uso de antimicrobianos en forma empírica.

\section{Material y Método}

En el Laboratorio de Microbiología del Hospital de Puerto Montt, Chile (hospital general de 400 camas), se revisaron los registros de todos los cultivos realizados entre los años 1995 y 2003, periodo en que la metodología de identificación y estudio de susceptibilidad se han mantenido uniformes.

Identificación de S pneumoniae. Las colonias morfológicamente sospechosas son estudiadas mediante susceptibilidad a optoquina en agar adicionado con $5 \%$ sangre de cordero e incubación por $18 \mathrm{~h}$ a $37^{\circ} \mathrm{C}$ en atmósfera con $3 \% \mathrm{CO} 2$. Los resultados dudosos (halos de inhibición entre 7 y $14 \mathrm{~mm}$ ) son confirmados mediante test de solubilidad biliar. Adicionalmente, las colonias sospechosas aisladas de líquidos normalmente estériles, son sometidas a un test de látex para S pneumoniae (BioMerieux).

Estudio de susceptibilidad a penicilina. Se realiza mediante antibiograma en difusión en agar Mueller Hinton adicionado con $5 \%$ sangre de cordero e incubación a $37^{\circ} \mathrm{C}$ con $3 \%$ CO2, utilizando sensidisco de oxacilina de $1 \mathrm{ug}$. A todas las cepas resistentes a oxacilina (halo de inhibición $\leq 19 \mathrm{~mm}$ ) se les determina la concentración inhibitoria mínima (CIM) mediante epsilometría (Etest) con tira de PNC y ceftriaxona. Para la interpretación de los resultados del antibiograma en difusión y de las CIM se utilizan las recomendaciones del National Committee for Clinical Laboratory Standards ${ }^{6}$.

Estadística. Los datos obtenidos fueron analizados usando el programa estadístico SPSS. Las variables categóricas fueron comparadas usando Chi cuadrado 0 el test exacto de Fisher cuando fue apropiado. Se consideró estadísticamente significativo un valor de $\mathrm{p}<0,05$.

\section{RESUlTADos}

Durante los 9 años de análisis, se identificaron 618 cepas de S pneumoniae, 407 aisladas en adultos $(65,9 \%)$ y 211 en población pediátrica $(34,1 \%)$. El 48,1\% (297/618) provenía de sitios normalmente estériles (cepas invasivas) (Tabla 1).

El 90,5\% de las cepas resultó sensible a PNC (CIM $<0,06 \mu \mathrm{g} / \mathrm{mL}$ ), 7,6\% de susceptibilidad intermedia (CIM 0,12-1 $\mu \mathrm{g} / \mathrm{mL}$ ) y 1,9\% resistente (CIM $\geq 2 \mu \mathrm{g} / \mathrm{mL}$ ).

En la Tabla 2 se muestra la distribución anual de las cepas con los porcentajes de susceptibilidad y resistencia correspondientes, observándose que en 9 años no ha existido una tendencia al aumento en la proporción de cepas con algún grado de resistencia a PNC. En la población pediátrica 87,7\% (185/211) de las cepas son sensibles y en los adultos 91,9\% (374/407)

\section{Tabla 1. O rigen de 297 cepas invasivas de Streptococcus pneumoniae}

\begin{tabular}{|lrr|}
\hline Sitio aislamiento & Número & Porcentaje \\
\hline Sangre & 172 & 57,9 \\
Líquido cefalorraquídeo & 54 & 18,2 \\
Líquido peritoneal & 32 & 10,8 \\
Líquido pleural & 24 & 8,1 \\
Osteoarticular & 8 & 2,6 \\
Otros & 7 & 2,4 \\
Total & 297 & 100,0 \\
\hline
\end{tabular}

Otros: absceso tubo-ovárico (2), absceso pulmonar (2), absceso SNC (2), retroperitoneal (1). 
Tabla 2. Susceptibilidad a Penicilina de 618 cepas de Streptococcus pneumoniae 1995-2003

\begin{tabular}{|c|c|c|c|c|}
\hline Año & $\begin{array}{c}\text { Sensible } \\
\mathrm{CIM} \leq 0,06 \mathrm{ug} / \mathrm{mL}\end{array}$ & $\begin{array}{c}\text { Intermedio } \\
\text { CIM } 0,12-1 \mathrm{ug} / \mathrm{mL}\end{array}$ & $\begin{array}{c}\text { Resistente } \\
\text { CIM } \geq 2 \mathrm{ug} / \mathrm{mL}\end{array}$ & Total \\
\hline 1995 & $73(86,9 \%)$ & $(7,1 \%)$ & $5 \quad(6,0 \%)$ & 84 \\
\hline 1996 & $56(91,8 \%)$ & $5 \quad(8,2 \%)$ & 0 & 61 \\
\hline 1997 & 50 (86,2\%) & $6 \quad(10,3 \%)$ & $(3,5 \%)$ & 58 \\
\hline 1998 & 72 (91,1\%) & $6 \quad(7,6 \%)$ & $1 \quad(1,3 \%)$ & 79 \\
\hline 1999 & $65(91,5 \%)$ & $6 \quad(8,4 \%)$ & 0 & 71 \\
\hline 2000 & $43(87,8 \%)$ & $5 \quad(10,2 \%)$ & $(2,0 \%)$ & 49 \\
\hline 2001 & $66(95,6 \%)$ & $3 \quad(4,3 \%)$ & 0 & 69 \\
\hline 2002 & $59(98,3 \%)$ & $1 \quad(1,7 \%)$ & 0 & 60 \\
\hline 2003 & $75(86,2 \%)$ & $9(10,3 \%)$ & $3 \quad(3,4 \%)$ & 87 \\
\hline Total & $559(90,5 \%)$ & $47 \quad(7,6 \%)$ & $12 \quad(1,9 \%)$ & 618 \\
\hline
\end{tabular}

(Tabla 3). La mayor resistencia en cepas pediátricas $(12,3 \%$ vs $8,1 \%)$ no es estadísticamente significativa $(p=0,112)$.

La diferencia entre la resistencia en las cepas invasivas comparada con las aisladas en sitios no estériles (Tabla 3) tampoco resultó significativa $(8,1 \%[24 / 297]$ vs $10,9 \%[35 / 321]),(p=0,273)$.

De las 12 cepas resistentes, sólo una tenía CIM de $4 \mu \mathrm{g} / \mathrm{mL}$ que correspondía a un adulto y el resto presentó CIM entre 1 y $2 \mu \mathrm{g} / \mathrm{mL}$.

\section{Discusión}

En este trabajo mostramos los resultados de la vigilancia de susceptibilidad de $\mathrm{S}$ pneumoniae a
PNC en el Hospital de Puerto Montt durante nueve años (1995-2003) con 9,5\% de cepas de susceptibilidad disminuida (CIM $\geq 0,12 \mu \mathrm{g} / \mathrm{mL}$ ) y sólo $1,9 \%$ de cepas con $\mathrm{CIM} \geq 2 \mu \mathrm{g} / \mathrm{mL}$.

El fenómeno de resistencia antimicrobiana, en especial para microorganismos de alta incidencia como S pneumoniae, constituye un problema de salud pública cuyas implicancias clínicas directas son, entre otras, la restricción de alternativas terapéuticas eficaces y el aumento de los costos de la atención en salud.

Las primeras publicaciones de resistencia aparecen en forma anecdótica en los años sesenta ${ }^{3}$ pero ya dos décadas más tarde, en diversos países aparecen publicaciones similares ${ }^{5,7}$ y posteriormente, hemos sido testigos de un sinnúmero de

Tabla 3. Susceptibilidad a penicilina de 618 cepas de $S$ pneumoniae según grupo etáreo y tipo de infección

\begin{tabular}{|lcccc|}
\hline & $\begin{array}{c}\text { Sensible } \\
\text { CIM } \leq 0,06 \mathrm{ug} / \mathrm{mL}\end{array}$ & $\begin{array}{c}\text { Intermedio } \\
\text { CIM 0,12-1 ug/mL }\end{array}$ & $\begin{array}{c}\text { Resistente } \\
\text { CIM } \geq 2 \mathrm{ug} / \mathrm{mL}\end{array}$ & Total \\
\hline Pediátricas & $185(87,7 \%)$ & $20(9,5 \%)$ & $6(2,8 \%)$ & 211 \\
Adultos & $374(91,9 \%)$ & $27(6,6 \%)$ & $6(1,5 \%)$ & 407 \\
Invasivas & $273(91,9 \%)$ & $20(6,7 \%)$ & $4(1,3 \%)$ & 297 \\
No invasivas & $286(89,1 \%)$ & $27(9,4 \%)$ & $8(2,5 \%)$ & 321 \\
\hline
\end{tabular}


comunicaciones que advierten de un incremento sostenido en la frecuencia de cepas con resistencia, llegando a cifras tan elevadas como las de España y Francia que bordean el 50\%4.

En Chile, las primeras publicaciones de S. pneumoniae con susceptibilidad disminuida a PNC aparecen en $1987^{\circ}$, en que de un total de 178 cepas, $21,9 \%$ mostraba CIM $\geq 0,12 \mu \mathrm{g} / \mathrm{mL}$. Más recientemente, en 1999, el Instituto de Salud Pública informó que en 945 cepas recibidas por esta entidad durante los años 1994 a 1998, la resistencia global fue $12,8 \%$ en pacientes mayores de 5 años y de 29,5\% en los menores de 5 años ${ }^{9}$. Esta publicación, que pudiera considerarse un reflejo de lo que ocurre en nuestro país, debe analizarse con cautela, ya que incluye principalmente cepas aisladas de sitios normalmente estériles y con evidentes diferencias de representación de las distintas regiones. Una publicación del Instituto Nacional del Tórax que analiza 75 cepas aisladas en tres periodos de los años 1997, 1998 y 1999, en población adulta, encontró una resistencia global de $16 \%$, incluyendo $5,3 \%$ de cepas con $\mathrm{CIM} \geq 2 \mu \mathrm{g} / \mathrm{mL}$ y concluye que en ese centro no se ha observado aumento en la frecuencia de cepas con resistencia en los tres años analizados ${ }^{10}$. En otro estudio, del Hospital de Niños Dr. Roberto del Río, en que se analizaron 78 cepas de S pneumoniae aisladas de infecciones invasivas durante los años 1994 a 1999, 35,9\% resultó con susceptibilidad disminuida a PNC y $14,1 \%$ de ellas con resistencia de alto nivel ${ }^{11}$. Se trata de una serie de cepas aisladas exclusivamente en población pediátrica ( $50 \%$ de los niños menores de 17 meses) donde se ha descrito mayor frecuencia de resistencia en S pneumoniae y en la que la alta frecuencia de cepas serotipo 14 y de cepas con $\mathrm{CIM} \geq 4 \mu \mathrm{g} / \mathrm{mL}$, sugiere cierta estructura clonal.

Nuestros resultados representan casi una década de vigilancia permanente del comportamiento de S pneumoniae frente a PNC y muestran bajos porcentajes de resistencia la que globalmente alcanza al 9,5\%. De las 618 cepas aisladas en estos nueve años, sólo 12 tuvieron $\mathrm{CIM} \geq 2 \mu \mathrm{g} / \mathrm{mL}$, lo que representa $1,9 \%$. No se observa una diferencia importante entre las cepas de adultos y pediátricas, con resistencia de $8,1 \%$ y $12,3 \%$, respectivamente. Sin embargo, este estudio muestra sólo la realidad de pacientes hospitalizados y no considera un cuadro de alta frecuencia en niños como es la
OMA, cuya principal etiología es S pneumoniae y cuyo manejo es habitualmente ambulatorio y sin estudio etiológico. Este aspecto debe ser considerado, ya que es en las cepas pediátricas donde se han descrito los porcentajes más elevados de resistencia, hecho que se ha evidenciado en las publicaciones mencionadas ${ }^{9-11}$, en las que la resistencia de las cepas aisladas en niños es casi el doble de la reportada en adultos. Sin embargo, este sesgo está presente en la mayoría de las publicaciones nacionales, por lo que desde ese punto de vista, nuestros resultados son comparables.

A diferencia de varios reportes internacionales ${ }^{12,13}$, en el curso de nueve años no hemos observado tendencia al aumento en la frecuencia de cepas con resistencia a PNC. Durante el año 2003, las cepas de susceptibilidad disminuida a la PNC alcanzaron a $13,7 \%$, cifra similar a la ya observada los años 2000 y 1997. Sin embargo, en los otros años analizados, el comportamiento de las cepas fue bastante homogéneo, con cifras de resistencia anuales tan bajas como $1,7 \%$ en 2002 , año en que sólo una de 60 cepas estudiadas presentó resistencia intermedia y no hubo cepas de alta resistencia. Podemos especular sobre las razones de estos resultados y postular una menor prevalencia de factores asociados a resistencia frente a PNC en S pneumoniae como el uso frecuente de antimicrobianos, especialmente betalactámicos y la concurrencia a sala cuna ${ }^{3}$, pero no disponemos de estudios poblacionales al respecto que pudieran explicar las diferencias con cepas aisladas en Santiago.

Las diferencias regionales en los índices de resistencia de S pneumoniae son una realidad, como lo ha demostrado el Proyecto Alexander iniciado en 1992 en Europa y Estados Unidos de Norteamérica y luego extendido a los 5 continentes $^{4}$ y que tiene como objetivo vigilar los niveles de resistencia de los principales agentes de infecciones respiratorias bajas adquiridas en la comunidad. Sus resultados han mostrado diferencias importantes entre países vecinos: mientras Italia y Suiza tienen resistencias del orden de 5\% y $9 \%$ respectivamente, Francia y España reportan cifras cercanas a $50 \%$. Estas diferencias regionales se verán, tal vez reflejadas con los primeros resultados de la Red Nacional de Vigilancia de Resistencia, implementada en Chile en 2002 por la Sociedad Chilena de Infectología en conjunto 
con el Instituto de Salud Pública ${ }^{14}$. Si esta iniciativa asegura la participación homogénea de centros de todas las regiones y utiliza metodología estandarizada, sujeta a controles de calidad internos y externos que permitan validar los datos obtenidos, será un gran aporte a la hora de formular guías terapéuticas locales para distintas patologías. Entre ellas, las infecciones del tracto respiratorio, de alta incidencia y de muy frecuente manejo empírico.

$\mathrm{Al}$ respecto, ha sido justamente la escasez de información nacional lo que ha determinado la utilización de guías terapéuticas internacionales en las decisiones clínicas individuales o la adaptación de las mismas en la formulación de guías nacionales. La norma para el manejo de la NAC elaboradas por la Sociedad de Enfermedades Respiratorias ${ }^{15}$, que actualmente se encuentra en revisión, incorporó la ceftriaxona como terapia de elección para la NAC que se hospitaliza, pero no consideró las distintas realidades que pueden existir en el país y que permiten avalar en algunas regiones como la nuestra, el uso de PNC en pacientes sin factores de riesgo para resistencia. Sumado a ello está el hecho

\section{REFERENCIAS}

1. American Academy of Pediatrics and American AcaDEMY of Family Physicians. Diagnosis and management of acute otitis media. Pediatrics 2004; 113: 1451-65.

2. Fine M, Smith M, Carson C, Mutha S, Sankey S, Weissfeld L et al. Prognosis and outcome of patients with community-acquired pneumonia: a meta-analysis. JAMA 1996; 275: 134-41.

3. Harmell J, Brown R. The drug-resistant Pneumococcus. Clinical relevance, therapy, and prevention. Chest 2000; 117: 530-41.

4. Adam D. Global antibiotic resistance in Streptococcus pneumoniae. J Antimicrob Chemother 2002; 50: 1-5.

5. Virk A, Steckelberg J. Clinical aspects of antimicrobial resistance. Mayo Clin Proc 2000; 75: 200-14.

6. National Committe for Clinical Laboratory StanDARDS. Performance standards for antimicrobial susceptibility testing: twelfth informational supplement 2002; 22: $\mathrm{N}^{\circ} 1$. de que si consideramos las distintas publicaciones nacionales, existen muy pocas cepas con CIM $\geq 4$ $\mu \mathrm{g} / \mathrm{mL}$, nivel en que el tratamiento con PNC no debiera considerarse, ya que las concentraciones tisulares alcanzadas, incluso con dosis altas, no permiten la erradicación de $\mathrm{S}$ pneumoniae ${ }^{16}$. El efecto que produce una norma es indudable y sin poner en duda la utilidad práctica de su formulación a nivel nacional, creemos que es necesario que en ellas se incorpore el criterio de las diferencias epidemiológicas locales, que debieran ser consideradas por el clínico a la hora de decidir una terapia antimicrobiana.

Es un hecho que la gran capacidad de adaptación bacteriana manifestada en la aparición y diseminación de resistencia, sobrepasa la velocidad con que la industria farmacológica puede desarrollar nuevos antimicrobianos y a la luz de estos alcances, creemos que una de las mejores y más eficaces estrategias para combatir este fenómeno, es el uso racional de los antimicrobianos. Conocer la realidad nacional y local, difundiendo en forma oportuna y permanente estos datos, puede contribuir a lograrlo.

7. TomasZ A. Antibiotic resistance in Streptococcus pneumoniae. Clin Infect Dis 1997; 24 suppl 1): 585-8.

8. Julet C, Giglo M, Camponovo R. Sensibilidad de Streptococcus pneumoniae y su importancia clínica. Rev Méd Chile 1987; 115: 852-7.

9. Heitmann I. Epidemiología nacional de las infecciones causadas por Streptococcus pneumoniae. Rev Chil Infect 1999; 16: 133-6.

10. Soler T, Salamanca L, Arbo G, Molna E. Estudio de sensibilidad in vitro de cepas de Streptococcus pneumoniae de infecciones respiratorias bajas en el Instituto Nacional del Tórax. Rev Méd Chile 2002; 130: 304-8.

11. Contreras L, Fica A, Figueroa C, Enríquez N, Urrutia P, HerRera P. Resistencia de Streptococcus pneumoniae y su asociación con factores clínicos y epidemiológicos. Rev Méd Chile 2002; 130: 26-34.

12. Doern G, Brueggemann A, Huynh H, Wingert E, RHOMBERG P. Antimicrobial resistance with Streptococcus pneumoniae in the United States, 1997-98. Emerg Infect Dis 1999; 5: 757-65. 
13. Pallares R, Leñares J, Vadilo $M$, Cabellos $C$, ManResa F, Viladrich P et al. Resistance to penicillin and cephalosporin and mortality from severa pneumococcal pneumonia in Barcelona, Spain. $\mathrm{N}$ Engl J Med 1995; 333: 474-80.

14. Valenzuela M, Prat S, Santolaya M. Implementación de una red nacional para la vigilancia de resistencia de agentes patógenos a antimicrobianos según síndromes clínicos. Rev Chil Infect 2003; 20: 119-25.
15. Sociedad Chilena de Enfermedades Respiratorias. Consenso nacional de neumonías adquiridas en la comunidad en adultos y niños. Rev Chil Enf Respir 1999; 15: 67-136.

16. Heffelfinger ZJ, Dowell S, Jorgensen J, Klugman K, Mabry L, Musher D et al. Management of community-acquired pneumonia in the era of pneumococcal resistance. Arch Intern Med 2000; 160: 1399-408. 